\title{
miR-21 increases the programmed cell death 4 gene-regulated cell proliferation in head and neck squamous carcinoma cell lines
}

\author{
ZHIFENG SUN ${ }^{1,2}$, SUPING LI ${ }^{2}$, ANDREAS M. KAUFMANN ${ }^{3}$ and ANDREAS E. ALBERS ${ }^{1}$ \\ ${ }^{1}$ Department of Otolaryngology, Head and Neck Surgery, Charité-Universitätsmedizin Berlin, Berlin, Germany; \\ ${ }^{2}$ Clinic for Gynecology, The Affiliated Hospital of Hubei Nationalities University, Enshi, Hubei, P.R. China; \\ ${ }^{3}$ Clinic for Gynecology, Charité-Universitätsmedizin Berlin, Berlin, Germany
}

Received July 2, 2014; Accepted August 6, 2014

DOI: $10.3892 /$ or.2014.3456

\begin{abstract}
MicroRNAs (miRs) are small non-coding RNAs that regulate the translation of many genes in normal and cancer cells where they are frequently dysregulated promoting tumor progression. Several studies have illustrated the potential of manipulating miR expression in cancer research and therapy. The aim of the present study was to investigate expression patterns of a panel of miRs in head and neck squamous cell carcinoma (HNSCC) shown to be relevant in other carcinomas and to elucidate their role if dysregulated. We performed analysis of miR-21, -200c, -138-1, -138-2, -25 and -34 expression by qRT-PCR in 6 HNSCC cell lines and computerized search for genetic targets of dysregulated miRNA-21 (miR-21). Lipofection of mock and anti-miR-21 and determination of expression efficiencies and final programmed cell death 4 (PDCD4) expression were carried out by luciferase assay and western blotting. MTT assay was used to measure cell proliferation and flow cytometry was performed for cell cycle analysis. Expression of miR-21 was most prominently upregulated in the HNSCC cell lines, particularly in UM-SCC11B (6.45 \pm 0.25 -fold, $\mathrm{P}<0.05)$ and UM-SCC9 (4.35 \pm 0.22 -fold, $\mathrm{P}<0.05)$ as compared to primary epidermal keratinocytes used as control. The expression levels of the other miRs showed no difference except for miR-34 and -138-1 each in one cell line. Subsequent transfection of precursor miR-21 stimulated proliferation while anti-miR-21 inhibited proliferation of both cell lines. PDCD4 was identified with software designed for this purpose as potential target gene of miR-21. Subsequently, its role in HNSCC lines was experimentally confirmed by regulation of PDCD4 transfecting miR-21 mimics and anti-miR-21. Finally, we showed that PDCD4 is negatively regulated by miR-21 at the post-transcriptional level via binding to the 3'-untranslated region of PDCD4 mRNA. A role of upregu-
\end{abstract}

Correspondence to: Dr Andreas E. Albers, Department of Otolaryngology, Head and Neck Surgery, Charité-Universitätsmedizin Berlin, Hindenburgdamm 30, D-12200 Berlin, Germany E-mail: andreas.albers@charite.de

Key words: miR-21, head and neck squamous cell carcinoma, programmed cell death 4 , dormancy lated miR-21 and reduced PDCD4 stimulating the proliferation was demonstrated in HNSCC lines and, in turn, transfection of anti-miR-21 upregulating PDCD4 reduced the cellular division rate. We explored miR-21 and PDCD4 expression as markers of progression and prognosis and for a potential translational value in the development of agents slowing growth of HNSCC and other carcinomas useful in palliative therapy or as a component of multi-modality treatments.

\section{Introduction}

Head and neck squamous cell carcinoma (HNSCC) is the sixth most common cancer worldwide. Despite ongoing improvements of established treatment modalities, the long-term survival rate of patients with HNSCC has improved only marginally over the past several decades. More than $60 \%$ of patients with advanced tumors or localized lymph node metastases succumb to the disease within five years after diagnosis (1).

MicroRNAs (miRs) are small non-coding RNAs that regulate the translation of many genes. Mature miRs are singlestranded, non-coding RNAs that are frequently dysregulated in cancer. miRs play key roles in various cellular processes, such as differentiation, cell growth, angiogenesis, epithelialto-mesenchymal transition (EMT) and invasion (2-4). Accumulating evidence suggests that a correlation between specific tumors and differential miR expression profiles exists (5-7), indicating specific molecular pathways activated in cancer cells during carcinogenesis and tumor progression. This insight stimulated an increasing number of studies analyzing miR expression profiles in different squamous carcinomas, including their potential clinical relevance $(8,9)$. Both messenger and non-coding RNAs can be detected in blood and studies indicate that miRs are particularly stable and abundant $(10,11)$. Circulating miRs could be derived from passive leakage from apoptotic or necrotic cancer cells yet also from normal tissue due to damage (e.g. trauma) or chronic inflammation $(12,13)$. In addition, both cancer and non-malignant cells, including immune cells, can actively release miRNAs, either microvesicle-associated, free or in a selective manner (14).

miRs not only regulate normal cellular development, they also play important roles in cancer development and progres- 
sion. Several studies reported that miRs can act either as oncogenes or tumor suppressors $(15,16)$. Others illustrated the potential of manipulating miR expression in cancer therapy. It is believed that miR-based therapeutic regulation of miRs has the potential to contribute to curing cancer, as they regulate whole programs of gene expression by suppressing hundreds of genes simultaneously (17).

For a few miRs, a functional relevance has already been demonstrated in HNSCC. miR-200c negatively modulates the expression of BMI1 and inhibits epithelial-mesenchymal transitions in malignant HNSCC (18). Ectopic transfection of miR-138 suppressed cell invasion and led to cell cycle arrest and apoptosis. Knockdown of miR-138 enhanced cell invasion and suppressed apoptosis, suggesting that miR-138 acts as a tumor suppressor and may serve as a therapeutic target for HNSCC patients at risk of metastasis (19). miR-34 coordinates with other miRs regulating signaling pathways, including the TGF-Wnt pathway, G1-S cell cycle progression, VEGF signaling pathway, apoptosis and survival pathways in nasopharyngeal carcinoma (20). In esophageal squamous cell carcinoma, miR-25 was upregulated significantly and correlated with the status of lymph node metastasis and TNM-classification. Overexpression of miR-25 markedly promoted migration and invasion of esophageal squamous cell carcinoma (21). miRNA-21 (miR-21), a well-known oncogenic miRNA, was found to be overexpressed in different types of human cancer, and it has been implicated in multiple malignancy-related processes including cell proliferation, apoptosis, invasion and metastasis $(22,23)$.

The present study clarified the role of a panel of miRs as potential drivers of biological aggressiveness in HNSCC. Exploring the expression patterns of these miRs, we noted in vitro a significant upregulation of miR-21 expression in HNSCC cell lines UM-SCC11B and UM-SCC9 which was different from the five other miRs tested (miR-200c, -138-1, $-138-2,-25$ and -34$)$. Using software specifically designed to identify miR target genes (miRanda and TargetScan), we found that miR-21 contains a consensus sequence to programmed cell death 4 (PDCD4), and identified PDCD4 as a potential target gene of miR-21. Therefore, we explored the biologic effects of miR-21 on PDCD4 in two distinct HNSCC cell lines.

PDCD4 is a recently-characterized tumor suppressor gene involved in the apoptotic machinery and in cell transformation and invasion, and tumor progression (24). PDCD4 protein expression is consistently downregulated in human cancer and cancer cell lines (24-27). Several mechanisms are involved in PDCD4 dysregulation; among others, the oncogenic miR-21 has been shown to specifically target the PDCD4 3'-untranslated region (3'-UTR), which negatively regulates PDCD4 expression.

The purpose of the present study was to explore a possible presence of the interdependence of miR-21 and PDCD4 in HNSCC. Consequently, we investigated and manipulated miR-21 and PDCD4-levels in two exemplary HNSCC lines.

\section{Materials and methods}

Cell culture. The HNSCC cell line panel was composed of UD-SCC-1 and -2 (provided by Dr Henning Bier, University of Düsseldorf), UM-SCC-9, -11B, -47, -104 (provided by
Dr Tom Carey, University of Michigan), of which UM-SCC47, -104 and UD-SCC-2 are HPV16-related. Normal primary epidermal keratinocytes (PCS-200-011) were purchased from American Type Culture Collection (ATCC, Manassas, VA, USA). All cells were cultured in DMEM or RPMI-1640 supplemented with $10 \%$ fetal bovine serum (both from HyClone, Logan, UT, USA) and $1 \%$ penicillin and streptomycin in a $5 \%$ $\mathrm{CO}_{2}$ atmosphere at $37^{\circ} \mathrm{C}$.

Quantitative real-time RT-PCR (qRT-PCR) detection of miRNA expression. qRT-PCR detection of miRs (miR-200c, $-21,-138-1,-138-2,-25$ and -34 ) was performed mostly as previously described (28). Total RNA was obtained from cell lines using the mirVana miRNA Isolation kit (Applied Biosystems, Foster City, CA, USA), according to the manufacturer's instructions. The expression of mature miR was determined by TaqMan real-time RT-PCR using the TaqMan miR assay (Applied Biosystems) and normalized using the $2^{-\Delta \Delta C T}$ method relative to U6-small nuclear RNA. All PCRs were carried out in triplicate.

Transfection with antisense oligonucleotides. The stabilityenhanced miR precursor that mimics miR-21 and the control non-specific miR precursor [pre-miR precursor, negative control (NC)] and the anti-miR-21 (miR-21 inhibitor) were purchased from Ambion (Ambion-Life Technologies, Darmstadt, Germany). The sequence for the miR-21 inhibitor was: 5'-UCAACAUCAGUCUGAUAAGCUA-3'; the sequences for miR-21 mimics were: 5'-UAGCUUAUCAGACUGAUGU UGA-3' and 5'-AACAUCAGUCUGAUAAGCUAUU-3'. The sequence for the NC miR inhibitor was: 5'-CAGUACUUUU GUGUAGUACAA-3'. Cells were trypsinized, counted and seeded onto 6-well plates the day prior to transfection to ensure $50 \%$ cell confluence on the day of transfection. Transfection of miR precursor/inhibitor into cells was performed using Lipofectamine 2000 (Invitrogen, Carlsbad, CA, USA) in accordance with the manufacturer's advised procedure. The $\mathrm{miR}$ precursor/inhibitor was used at a final concentration of $100 \mathrm{nM}$. Post-transfection, real-time RT-PCR, western blotting and cell proliferation analysis were performed. The transfection efficiency was assessed by fluorescence microscope (nuclei were stained with DAPI). Nearly all cells exhibited Cy3 staining, indicating that the miR-21 precursor/ inhibitor and controls were effectively transfected into UM-SCC9 cells.

Western blot analysis. The transfected cells and untreated control cells were isolated $72 \mathrm{~h}$ after transfection and proteins were extracted in a solution of RIPA and Halt ${ }^{\mathrm{TM}}$ Protease Inhibitor Cocktail (Thermo Scientific, Waltham, MA, USA) from cells and subjected to SDS-polyacrylamide gel electrophoresis. Quantification of total protein was carried out by bicinchoninic acid (BCA) (Sigma, St. Louis, MO, USA). The proteins $(100 \mu \mathrm{g})$ were subjected to $12 \%$ SDS-polyacrylamide gel electrophoresis. Separated proteins were electrophoretically transferred to nitrocellulose (NC) membrane (Bio-Rad, Hercules, CA, USA) and immunoblotted with anti-human PDCD4 polyclonal (1:1,000; Covance, Princeton Township, NJ, USA), anti-GAPDH (1:5,000; Sigma). Immunoreactive proteins were visualized using the Odyssey Infrared Imaging 

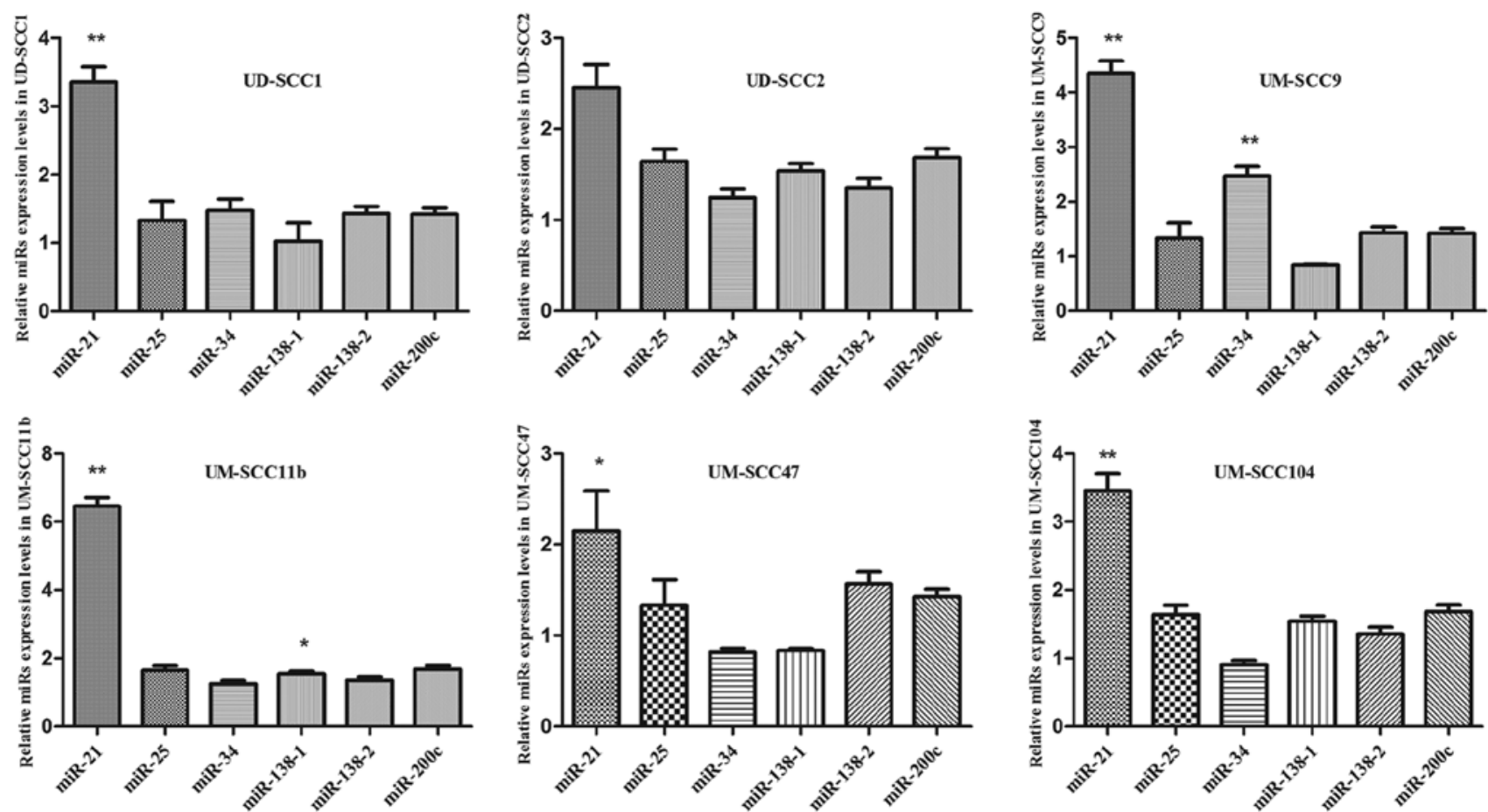

Figure 1. Upregulation of miR-21 expression in HNSCC cell lines. qRT-PCR for miRs was performed using 6 HNSCC cell lines including two HPV16-associated lines (UM-SCC47 and -104) and one normal cell line as described in Materials and methods. The mean and standard deviation of miR expression levels relative to the U6-small nuclear RNA expression level compared with the normal cell line is shown. The data represent triplicate experimental measurements ("P $<0.05$, $\left.{ }^{* *} \mathrm{P}<0.01\right)$. HNSCC, head and neck squamous cell carcinoma

System (LI-COR, Lincoln, NE, USA), as described by the manufacturer.

Cell proliferation assay. A diphenyltetrazolium bromide (MTT) assay was performed to determine the cell proliferation. Five thousand cells were seeded to each well of a 96 -well plate and grown for 24, 48, 72 and $96 \mathrm{~h}$. Next, the medium was removed and cells were washed with phosphate-buffered saline (PBS). Then $5 \mathrm{~g} / \mathrm{l}$ of thiazolyl tetrazolium (Ameresco, Indianapolis, IN, USA) was added to each well. After an additional $4 \mathrm{~h}$ of incubation, MTT was removed and $150 \mu \mathrm{l}$ of dimethyl sulfoxide (Sigma) was added. The viability of the cells was calculated from the absorption at $570 / 630 \mathrm{~nm}$ with an enzyme-linked immunosorbent assay reader. The experiment was repeated three times.

Cell cycle analysis. At $48 \mathrm{~h}$ post-transfection with the miR-21 precursor/inhibitor or control precursor $(100 \mathrm{nM})$, cells, including untreated and mock controls, were collected by trypsinization and washed with PBS. For cell cycle analysis, the cells were fixed with $75 \%$ ethanol and stored at $4{ }^{\circ} \mathrm{C}$ overnight. The following day, fixed cells were washed with PBS, treated with RNase A $(50 \mu \mathrm{g} / \mathrm{ml})$, and stained with propidium iodide (PI) $(50 \mu \mathrm{g} / \mathrm{ml})$ for $30 \mathrm{~min}$ in the dark. The stained cells were analyzed by flow cytometry (FACSCalibur; Becton-Dickinson, Franklin Lakes, NJ, USA). Cellular debris and fixation artifacts were removed by an exclusion gate and the cell populations in $\mathrm{G} 0 / \mathrm{G} 1, \mathrm{~S}$ and $\mathrm{G} 2 / \mathrm{M}$ phases were quantified using FlowJo 7.6.2 software (Tree Star, Ashland, OR, USA). At least 10,000 cells for each condition were analyzed to obtain a reliable signal.
Luciferase assays. The full-length 3'-UTR of PDCD4 mRNA containing the miR-21 binding site was amplified by PCR primers: 5'-ggggagctcatataagaactcttgcagtct-3' (forward) and 5'-gggaagcttggtgtacattcttctagaac-3' (reverse), and cloned into the SacI-HindIII site of the pMIR-REPORT kit (Applied Biosystems) and termed Luc-PDCD4-Wt. To generate miR-21 binding site deletion mutants, the seed sequences were deleted using the QuikChange Site-Directed Mutagenesis kit (Agilent Technologies, La Jolla, CA, USA), with Luc-PDCD4-Wt as a template. The resulting mutant was termed Luc-PDCD4-d. For the reporter assays, the cells were transiently transfected with the luciferase vector as control vector, and either anti-miR-21 oligonucleotide or negative control using Lipofectamine 2000. Reporter assays were performed 24-h post transfection using the Luciferase Assay kit (Promega, Madison, WI, USA). $\beta$-galactosidase activity was used for normalizing the transfection efficiency.

Statistical analysis. Data are shown as means \pm SD and subjected to one-way analysis of variance with factors of treatment using the SPSS 13.0 for Windows (SPSS, Chicago, IL, USA). Comparisons between two groups were performed by an unpaired Student's t-test. $\mathrm{P}<0.05$ (one-tailed) was considered to indicate a statistically significant result.

\section{Results}

The expression of miR-21 is upregulated in HNSCC lines. To explore the role of miRNAs in HNSCC, detecting expression levels of different miRNAs was a primary consideration. Expression of miR-21, -200c, -138-1, -138-2, -25 and -34 


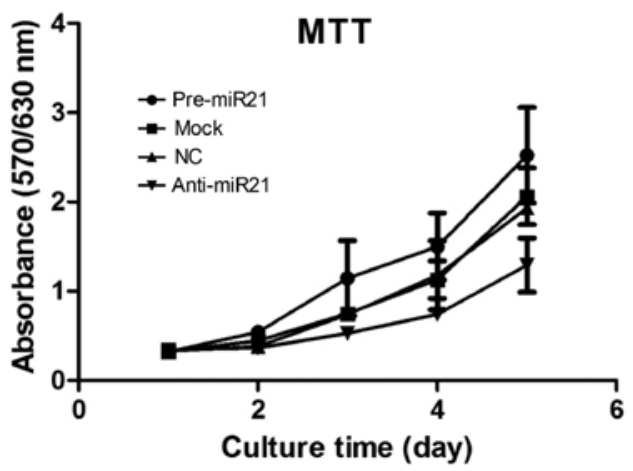

Figure 2. The effect of miR-21 on the proliferation of UM-SCC9 cells. Cell proliferation was measured by MTT assay. UM-SCC9 cells were transfected with miR-21 precursor/inhibitor or control at a final concentration of $100 \mathrm{nM}$ and, at 24-h post-transfection, the MTT assay was performed every $24 \mathrm{~h}$ for 4 days. Results are the mean of three independent experiments and are shown as mean $\pm \mathrm{SD}(\mathrm{P}<0.05)$.

were assessed by qRT-PCR detection in 6 HNSCC lines and compared to normal primary epidermal keratinocytes as control. The cell lines chosen were derived from human HNSCC, from patients exhibiting an aggressive clinical course who developed local or regional recurrence, and who had died within two years of diagnosis (29). Moreover, with regard to the etiology of HNSCC, three HPV-related (UM-SCC-47, -104 and UD-SCC-2) cell lines were included. qRT-PCR revealed different levels of expression of the tested miRs in those HNSCC cells (Fig. 1). miR-21 exhibited the highest expres- sion in the tested HNSCC cell lines, with respect to normal primary epidermal keratinocytes used for control purposes. This observation has not been previously described. From the other tested miRs, only miR-34 showed a significant upregulation in UM-SCC9 and miR-138-1 in UM-SCC11B. Thus, further investigations were carried out to determine the role of miR-21 in HNSCC.

miR-21 regulates the growth of HNSCC cells. Considering the marked upregulation of miR-21 in the HNSCC lines, it was hypothesized that it may function as a tumor promoter. This hypothesis was supported by results from computational analyses indicating interactions between miR-21 and the tumor-suppressor PDCD4 as described below. Consequently, we focused on proof of principle by testing the effect of miR-21 on the growth of one exemplary cell line (UM-SCC9). In an MTT proliferation assay, cells transfected with a miR-21 precursor grew more rapidly than the mock control (Fig. 2A and B). The difference in the proliferative activity of transfected cells indicated that overexpression of miR-21 promotes growth activity in both HNSCC lines. Nevertheless, inhibition of miR-21 by anti-miR-21 resulted in growth delay (Fig. 2A and B). These results suggest that miR-21 promotes cell growth, and disturbance of miR-21 can effectively restrain the proliferation of HNSCC cells in vitro.

Downregulation of miR-21 expression induces cell cycle arrest in Gl/S phase. To elucidate the mechanism of miR-21-mediated cell growth of HNSCC cells (UM-SCC9), cell cycle analysis of
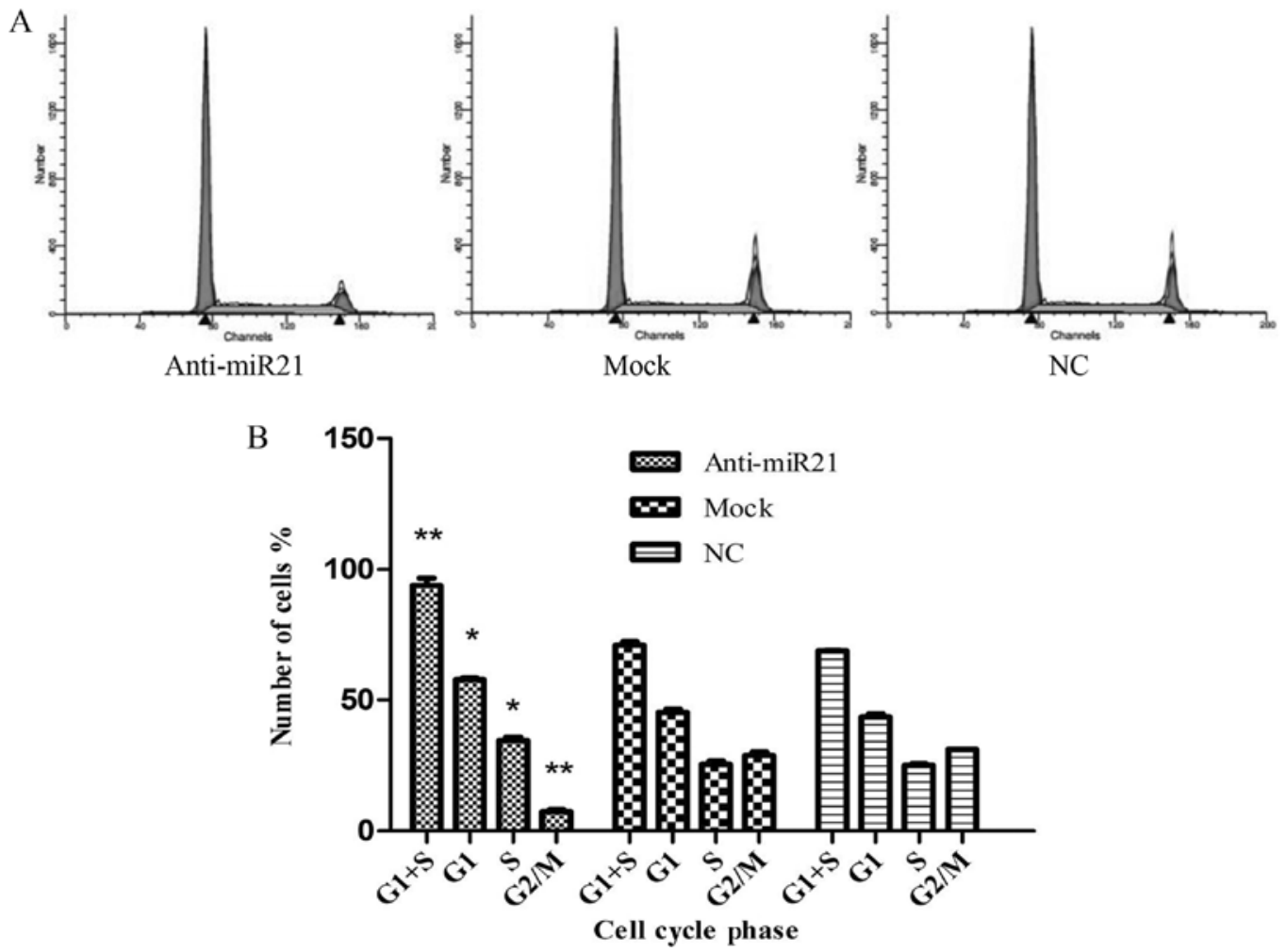

Figure 3. The effect of miR-21 on cell cycle distribution of UM-SCC9 cells. (A) Representative histograms of DNA content in anti-miR12 transfected UM-SCC9 cells compared to mock-transfected and normal controls. Cells were stained with PI and analyzed by flow cytometry $48 \mathrm{~h}$ post-transfection. (B) Analysis of proportions of cells in different phases of the cell cycle. The results are the mean of three independent experiments and shown as mean \pm SD $(\mathrm{P}<0.05)$. PI, propidium iodide. 
A

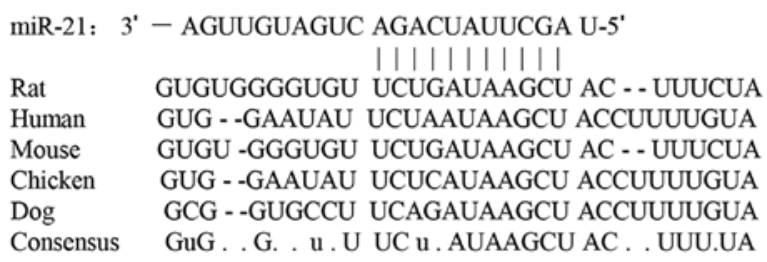

B

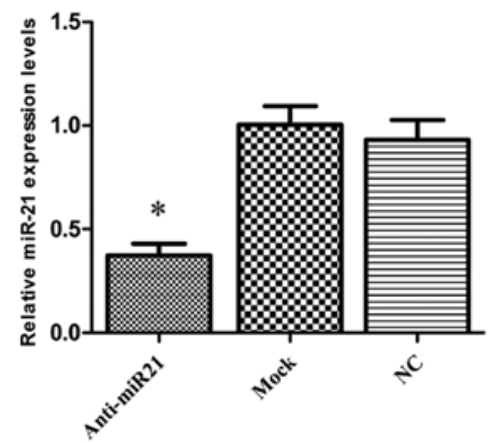

C

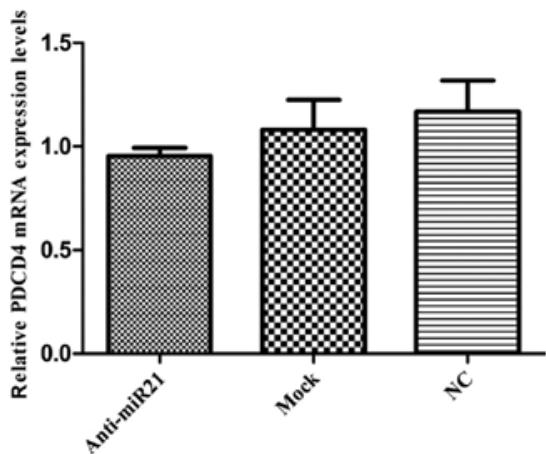

D
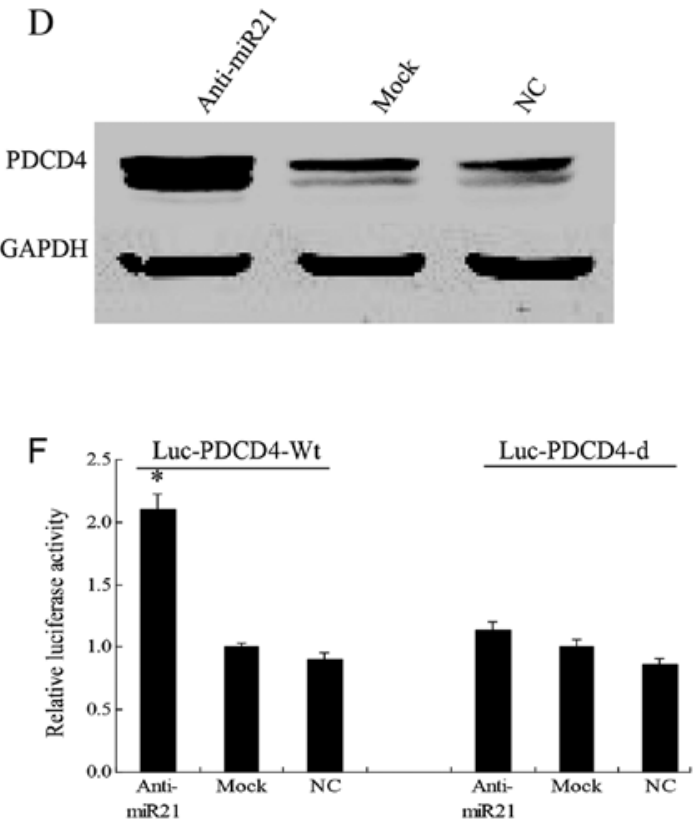

Figure 4. miR-21 regulates the PDCD4 expression by targeting the 3'-untranslated region (3'-UTR) of PDCD4. (A) Computational analysis indicated that PDCD4 is a potential target gene of miR-21. The sequences for the binding sites in the 3'UTRs of PDCD4 are highly conserved among different species. (B) Quantification of miR-21 expression by qRT-PCR. "P<0.05. (C) PDCD4 mRNA expression analysis by qRT-PCR. PDCD4 expression levels were normalized to GAPDH expression. (D) Western blot analysis of PDCD4 protein expression. (E) The construction of full-length 3'-UTR of PDCD4 cDNA containing a miR-21-binding site for the reporter plasmid. The seed sequences are underlined. (F) Relative expression levels of Luc-PDCD4-Wt and Luc-PDCD4-d in anti-miR-21, mock and normal controls. The results were normalized to the transfection efficiency using the $\beta$-galactosidase activity. PDCD4, programmed cell death 4.

anti-miR-21 cells was performed (Fig. 3A). The results demonstrated that, when compared with the mock and normal control group, the percentage of anti-miR-21-transfected UM-SCC9 cells in $\mathrm{G} 1 / \mathrm{S}$ phase increased from $71.03 \pm 1.26$ to $93.93 \pm 2.67 \%$ $(\mathrm{P}<0.05)$, whereas the percentage of cells in $\mathrm{G} 2 / \mathrm{M}$ phase decreased from $28.96 \pm 1.26$ to $7.4 \pm 0.79 \%(\mathrm{P}<0.05)$ (Fig. 3B). These results indicate that downregulation of miR-21 expression induces G1/S phase arrest, thereby confirming the stimulating role of miR-21 for cell division.

miR-21 PDCD4 expression by targeting the PDCD4-3'-UTR. Computational analysis with specifically designed software indicated that PDCD4 is a potential target gene of miR-21 (Fig. 4A). The sequences for the binding sites in the 3'UTRs of PDCD4 are highly conserved among different species. Previous reports demonstrated that there was a significant inverse correlation between miR-21 expression and PDCD4 protein levels $(30,31)$, and low expression levels of PDCD4 in primary HNSCC were also found (32). If PDCD4 was regulated by miR-21 as hypothesized, a miR-21 inhibitor should increase its expression in HNSCC. To test this hypothesis,
UM-SCC9 cells were used to determine in vitro whether the suppression of miR-21 also affected PDCD4 expression in HNSCC cells. The downregulation of endogenous miR-21 with anti-miR-21 (Fig. 4B) led to a significant increase in PDCD4 protein (Fig. 4D) without any change in PDCD4 mRNA levels (Fig. 4C). Thus, the results suggested that PDCD4 is a potential miR-21 target gene. Next, to further confirm that miR-21 is able to directly bind to PDCD4 and inhibit PDCD4 expression and to determine whether the 3'-UTR of PDCD4 mRNA is a functional target of miR-21, a reporter plasmid driven by the SV40 basal promoter, harboring the full-length 3'-UTR of PDCD4 mRNA at the 3' position of the luciferase reporter gene, was cloned (Fig. 4E). The transient transfection of UM-SCC9 cells with the reporter plasmid and anti-miR-21 inhibitor led to a significant increase of reporter activity in comparison with the negative control (Fig. 4F). However, the activity of the reporter construct deleted at the seed sequences of miR-21 target site was unaffected by a simultaneous transfection with anti-miR-21 (Fig. 4F). These results indicate that miR-21 regulates PDCD4 expression at the post-transcriptional level by targeting the PDCD4-3'-UTR. 


\section{Discussion}

In the present study, we are first to show that miR-21 regulates cellular proliferation in HNSCC lines. We demonstrated that the tumor suppressor PDCD4 is negatively regulated by miR-21 at the post-transcriptional level via binding to the 3'-untranslated region (3'-UTR) of PDCD4 mRNA.

miRs regulate a variety of cellular pathways through the regulation of the expression of multiple target genes (33). In this regard, miR-21 has been suggested to function as an oncogene, since it is overexpressed in many types of solid malignancy (6), particularly in malignancies such as breast cancer (34), glioblastoma (35), prostate (36), ovarian (37), pancreatic (38), colon (39) and gastric cancer (40), cholangiocarcinoma (41), hepatocellular cancer (42), HNSCC (43) and esophageal cancer (44). Furthermore, an association between $\mathrm{miR}-21$ expression and prognosis has been proposed in pancreatic cancer and colon adenocarcinoma $(45,46)$. In the present study, miR-21 expression in HNSCC was significantly higher than that of matched normal epithelium, and PDCD4 protein correlated inversely with miR-21 level as shown in previous studies $(43,47)$. Whether it may serve as a prognostic factor in HNSCC as observed in other malignancies remains, to date, unknown $(35,42,48-51)$. In the present study, we sought to elucidate its function as a regulator. The tumor suppressor gene PDCD4 was originally characterized as an inhibitor of cellular transformation in a mouse cell culture model (52). PDCD4 expression is downregulated or lost in several tumor types (53) and ectopic expression of PDCD4 reduces tumor formation in a mouse skin cancer model (54). On a molecular level, PDCD4 binds and inhibits the translation initiation factor eukaryotic initiation factor 4a, thereby impacting protein translation (55). In addition, PDCD4 has been found to inhibit activator protein-mediated transactivation (56) and to induce the expression of the cyclin-dependent kinase inhibitor p21 (57). As a result, the loss of PDCD4 confers growth advantages to the cells by several means, thereby facilitating the development and promotion of cancer. In recent studies, PDCD4 was reported as a functional target of miR-21 in various aspects of tumor progression; cell proliferation, invasion, metastasis and neoplastic transformation in breast cancer (58) and invasion, intravasation, and metastasis in colon cancer (47). In the present study, a high expression of miR-21 was found in the 6 tested HNSCC lines of which two were HPV-associated. Furthermore, suppression of miR-21 in vitro led to reduction of cellular proliferation. Therefore, we hypothesized that PDCD4 was also an important target of miR-21 in HNSCC. As shown in Fig. 4D, anti-miR-21-transfected cells showed a significant increase in PDCD4 protein without any change in PDCD4 mRNA. Transient transfection of cells with a reporter plasmid containing the 3'-UTR of PDCD4 mRNA and antimiR-21 inhibitor led to a significant increase of reporter activity. These findings suggest that the PDCD4 is negatively regulated by miR-21 at the post-translational level via binding to the 3'-UTR of PDCD4 mRNA.

In the present study, we also found that downregulation of miR-21 expression induced cell cycle arrest in G1/S phase. The actions of some cytotoxic drugs are cell cycle-specific. The $\mathrm{S}$ phase is where DNA synthesis takes place. Many cell cyclespecific drugs act only on cells that are in the $\mathrm{S}$ phase. These drugs interfere with DNA synthesis in some way, therefore miR-21 may play a role in increasing the sensitivity of HNSCC lines to these cell cycle-specific cytotoxic drugs.

In summary, miR-21 was overexpressed in all tested HNSCC cell lines, including two HPV-associated lines, and anti-miR-21 inhibited cellular proliferation in vitro. These effects are possibly due to downregulation of the tumor suppressor PDCD4 by miR-21. These findings raise the possibility that anti-miR-21 may have potential therapeutic value in HNSCC patients and may also play a role as predictor in healthy individuals. It has been shown that anti-miR oligonucleotides could stay for a relatively long period of time in animals $(59,60)$. Therefore, miRs, in particular miR-21, may serve as a potentially useful target for cancer therapy.

\section{References}

1. Jemal A, Bray F, Center MM, Ferlay J, Ward E and Forman D: Global cancer statistics. CA Cancer J Clin 61: 69-90, 2011.

2. Cheng ZX, Sun B, Wang SJ, et al: Nuclear factor- $\kappa \mathrm{B}-$ dependent epithelial to mesenchymal transition induced by HIF-1 $\alpha$ activation in pancreatic cancer cells under hypoxic conditions. PLoS One 6: e23752, 2011

3. Semenza GL: HIF-1 and tumor progression: pathophysiology and therapeutics. Trends Mol Med 8 (Suppl 4): S62-S67, 2002.

4. Imai T, Horiuchi A, Wang C, et al: Hypoxia attenuates the expression of E-cadherin via up-regulation of SNAIL in ovarian carcinoma cells. Am J Pathol 163: 1437-1447, 2003.

5. Lu J, Getz G, Miska EA, et al: MicroRNA expression profiles classify human cancers. Nature 435: 834-838, 2005.

6. Volinia S, Calin GA, Liu CG, et al: A microRNA expression signature of human solid tumors defines cancer gene targets. Proc Natl Acad Sci USA 103: 2257-2261, 2006.

7. Rosenfeld N, Aharonov R, Meiri E, et al: MicroRNAs accurately identify cancer tissue origin. Nat Biotechnol 26: 462-469, 2008.

8. Chu Y, Zhu H, Lv L, Zhou Y and Huo J: MiRNAs in oesophageal squamous cancer. Neth J Med 71: 69-75, 2013.

9. Janiszewska J, Szaumkessel M and Szyfter K: microRNAs are important players in head and neck carcinoma: a review. Crit Rev Oncol Hematol 88: 716-728, 2013.

10. Wu Q, Wang C, Lu Z, Guo L and Ge Q: Analysis of serum genome-wide microRNAs for breast cancer detection. Clin Chim Acta 413: 1058-1065, 2012.

11. Liang H, Gong F, Zhang S, Zhang CY, Zen K and Chen X: The origin, function, and diagnostic potential of extracellular microRNAs in human body fluids. Wiley Interdiscip Rev RNA 5: 285-300, 2014.

12. Endo K, Weng H, Kito N, Fukushima Y and Iwai N: miR-216a and miR-216b as markers for acute phased pancreatic injury. Biomed Res 34: 179-188, 2013.

13. Olivieri F, Rippo MR, Procopio AD and Fazioli F: Circulating inflamma-miRs in aging and age-related diseases. Front Genet 4: 121,2013

14. de Yébenes VG, Bartolomé-Izquierdo N and Ramiro AR: Regulation of B-cell development and function by microRNAs. Immunol Rev 253: 25-39, 2013.

15. Stefani G: Roles of microRNAs and their targets in cancer. Expert Opin Biol Ther 7: 1833-1840, 2007.

16. Yang J, Hao Y and Xi JJ: Therapeutic application of microRNAs against human cancers. J Lab Autom 18: 30-33, 2013.

17. Farazi TA, Spitzer JI, Morozov P and Tuschl T: miRNAs in human cancer. J Pathol 223: 102-115, 2011.

18. Lo WL, Yu CC, Chiou GY, et al: MicroRNA-200c attenuates tumour growth and metastasis of presumptive head and neck squamous cell carcinoma stem cells. J Pathol 223: 482-495, 2011.

19. Liu X, Jiang L, Wang A, Yu J, Shi F and Zhou X: MicroRNA-138 suppresses invasion and promotes apoptosis in head and neck squamous cell carcinoma cell lines. Cancer Lett 286: 217-222, 2009.

20. Chen HC, Chen GH, Chen YH, et al: MicroRNA deregulation and pathway alterations in nasopharyngeal carcinoma. Br J Cancer 100: 1002-1011, 2009.

21. Xu X, Chen Z, Zhao X, et al: MicroRNA-25 promotes cell migration and invasion in esophageal squamous cell carcinoma. Biochem Biophys Res Commun 421: 640-645, 2012. 
22. Gao W, Xu J, Liu L, Shen H, Zeng H and Shu Y: A systematicanalysis of predicted miR-21 targets identifies a signature for lung cancer. Biomed Pharmacother 66: 21-28, 2012.

23. Schee K, Boye K, Abrahamsen TW, Fodstad Ø and Flatmark K: Clinical relevance of microRNA miR-21, miR-31, miR-92a miR-101, miR-106a and miR-145 in colorectal cancer. BMC Cancer 12: 505, 2012.

24. Young MR, Santhanam AN, Yoshikawa N and Colburn NH: Have tumor suppressor PDCD4 and its counteragent oncogenic miR-21 gone rogue? Mol Interv 10: 76-79, 2010.

25. Allgayer $\mathrm{H}: \mathrm{Pdcd} 4$, a colon cancer prognostic that is regulated by a microRNA. Crit Rev Oncol Hematol 73: 185-191, 2010.

26. Fassan M, Pizzi M, Battaglia G, et al: Programmed cell death 4 (PDCD4) expression during multistep Barrett's carcinogenesis. J Clin Pathol 63: 692-696, 2010.

27. Fassan M, Pizzi M, Giacomelli L, et al: PDCD4 nuclear loss inversely correlates with miR-21 levels in colon carcinogenesis. Virchows Arch 458: 413-419, 2011.

28. Chen C, Ridzon DA, Broomer AJ, et al: Real-time quantification of microRNAs by stem-loop RT-PCR. Nucleic Acids Res 33: e179, 2005

29. Wolf JS, Chen Z, Dong G, et al: IL (interleukin)-1 $\alpha$ promotes nuclear factor- $\kappa \mathrm{B}$ and AP-1-induced IL-8 expression, cell survival, and proliferation in head and neck squamous cell carcinomas. Clin Cancer Res 7: 1812-1820, 2001.

30. Itani S, Kunisada T, Morimoto Y, et al: MicroRNA-21 correlates with tumorigenesis in malignant peripheral nerve sheath tumor (MPNST) via programmed cell death protein 4 (PDCD4). J Cancer Res Clin Oncol 138: 1501-1509, 2012.

31. Horiuchi A, Iinuma H, Akahane T, Shimada R and Watanabe $T$ : Prognostic significance of PDCD4 expression and association with microRNA-21 in each Dukes' stage of colorectal cancer patients. Oncol Rep 27: 1384-1392, 2012.

32. Wang J and Zhang Y: Expression of programmed cell death 4 protein is closely correlated with laryngeal squamous cell carcinomas. Lin Chung Er Bi Yan Hou Tou Jing Wai Ke Za Zhi 25: 539-541, 2011 (In Chinese).

33. Bartel DP: MicroRNAs: genomics, biogenesis, mechanism, and function. Cell 116: 281-297, 2004.

34. Iorio MV,Ferracin M, Liu CG, et al: MicroRNA gene expression deregulation in human breast cancer. Cancer Res 65: 7065-7070, 2005 .

35. Chan JA, Krichevsky AM and Kosik KS: MicroRNA-21 is an antiapoptotic factor in human glioblastoma cells. Cancer Res 65: 6029-6033, 2005.

36. Shi GH, Ye DW, Yao XD, et al: Involvement of microRNA-21 in mediating chemo-resistance to docetaxel in androgen-independent prostate cancer PC3 cells. Acta Pharmacol Sin 31: 867-873, 2010.

37. Xu YZ, Xi QH, Ge WL and Zhang XQ: Identification of serum microRNA-21 as a biomarker for early detection and prognosis in human epithelial ovarian cancer. Asian Pac J Cancer Prev 14: $1057-1060,2013$

38. Giovannetti E, Funel N, Peters GJ, et al: MicroRNA-21 in pancreatic cancer: correlation with clinical outcome and pharmacologic aspects underlying its role in the modulation of gemcitabine activity. Cancer Res 70: 4528-4538, 2010.

39. Feng YH, Wu CL, Tsao CJ, et al: Deregulated expression of sprouty2 and microRNA-21 in human colon cancer: correlation with the clinical stage of the disease. Cancer Biol Ther 11: 111-121, 2011.

40. Zhang BG, Li JF, Yu BQ, Zhu ZG, Liu BY and Yan M: microRNA-21 promotes tumor proliferation and invasion in gastric cancer by targeting PTEN. Oncol Rep 27: 1019-1026, 2012.

41. Selaru FM, Olaru AV, Kan T, et al: MicroRNA-21 is overexpressed in human cholangiocarcinoma and regulates programmed cell death 4 and tissue inhibitor of metalloproteinase 3. Hepatology 49: 1595-1601, 2009.
42. Meng F, Henson R, Wehbe-Janek H, Ghoshal K, Jacob ST and Patel T: MicroRNA-21 regulates expression of the PTEN tumor suppressor gene in human hepatocellular cancer. Gastroenterology 133: 647-658, 2007.

43. Tran N, McLean T, Zhang X, et al: MicroRNA expression profiles in head and neck cancer cell lines. Biochem Biophys Res Commun 358: 12-17, 2007.

44. Huang S, Li XQ, Chen X, Che SM, Chen W and Zhang XZ: Inhibition of microRNA-21 increases radiosensitivity of esophageal cancer cells through phosphatase and tensin homolog deleted on chromosome 10 activation. Dis Esophagus 26: 823-831, 2013.

45. Schetter AJ, Leung SY, Sohn JJ, et al: MicroRNA expression profiles associated with prognosis and therapeutic outcome in colon adenocarcinoma. JAMA 299: 425-436, 2008.

46. Dillhoff M, Liu J, Frankel W, Croce C and Bloomston M: MicroRNA-21 is overexpressed in pancreatic cancer and a potential predictor of survival. J Gastrointest Surg 12: 2171-2176, 2008.

47. Asangani IA, Rasheed SA, Nikolova DA, et al: MicroRNA-21 (miR-21) post-transcriptionally downregulates tumor suppressor Pdcd4 and stimulates invasion, intravasation and metastasis in colorectal cancer. Oncogene 27: 2128-2136, 2008.

48. Feber A, Xi L, Luketich JD, et al: MicroRNA expression profiles of esophageal cancer. J Thorac Cardiovasc Surg 135: 255-260, 2008.

49. Fulci V, Chiaretti S, Goldoni M, et al: Quantitative technologies establish a novel microRNA profile of chronic lymphocytic leukemia. Blood 109: 4944-4951, 2007.

50. Iorio MV, Visone R, Di Leva G, et al: MicroRNA signatures in human ovarian cancer. Cancer Res 67: 8699-8707, 2007.

51. Markou A, Tsaroucha EG, Kaklamanis L, Fotinou M, Georgoulias V and Lianidou ES: Prognostic value of mature microRNA-21 and microRNA-205 overexpression in non-small cell lung cancer by quantitative real-time RT-PCR. Clin Chem 54: 1696-1704, 2008

52. Yang HS, Jansen AP, Nair R, et al: A novel transformation suppressor, Pdcd4, inhibits AP-1 transactivation but not NF- $\kappa$ B or ODC transactivation. Oncogene 20: 669-676, 2001.

53. Jansen AP, Camalier CE, Stark C and Colburn NH: Characterization of programmed cell death 4 in multiple human cancers reveals a novel enhancer of drug sensitivity. Mol Cancer Ther 3: 103-110, 2004

54. Jansen AP, Camalier CE and Colburn NH: Epidermal expression of the translation inhibitor programmed cell death 4 suppresses tumorigenesis. Cancer Res 65: 6034-6041, 2005.

55. Göke A, Göke R, Knolle A, et al: DUG is a novel homologue of translation initiation factor $4 \mathrm{G}$ that binds eIF4A. Biochem Biophys Res Commun 297: 78-82, 2002.

56. Yang HS, Jansen AP, Komar AA, et al: The transformation suppressor Pded4 is a novel eukaryotic translation initiation factor 4A binding protein that inhibits translation. Mol Cell Biol 23: 26-37, 2003.

57. Göke R, Barth P, Schmidt A, Samans B and Lankat-Buttgereit B: Programmed cell death protein 4 suppresses CDK $1 / \mathrm{cdc} 2$ via induction of $\mathrm{p} 21^{\text {Waf1/Cip1 }}$. Am J Physiol Cell Physiol 287: C1541-C1546, 2004.

58. Frankel LB, Christoffersen NR, Jacobsen A, Lindow M, Krogh A and Lund AH: Programmed cell death 4 (PDCD4) is an important functional target of the microRNA $m i R-21$ in breast cancer cells. J Biol Chem 283: 1026-1033, 2008.

59. Xu L, Dai WQ, Xu XF, Wang F, He L and Guo CY: Effects of multiple-target anti-microRNA antisense oligodeoxyribonucleotides on proliferation and migration of gastric cancer cells. Asian Pac J Cancer Prev 13: 3203-3207, 2012.

60. Lennox KA and Behlke MA: A direct comparison of antimicroRNA oligonucleotide potency. Pharm Res 27: 1788-1799, 2010. 